\title{
BULK MICROMACHINED TITANIUM MICROMIRROR DEVICE WITH SLOPING ELECTRODE GEOMETRY
}

\author{
Masa P. Rao ${ }^{1}$, Marco. F. Aimi ${ }^{2}$, and Noel C. MacDonald ${ }^{1,2}$ \\ ${ }^{1}$ Mechanical \& Environmental Engineering Department, University of California, Santa Barbara (UCSB), Santa \\ Barbara, CA 93106 \\ ${ }^{2}$ Materials Department, University of California, Santa Barbara (UCSB), Santa Barbara, CA 93106
}

\begin{abstract}
This paper reports the fabrication of a bulk micromachined hybrid torsional micromirror device composed of titanium mirror structures bonded to an underlying silicon sloping electrode structure. The fabrication of the device is enabled by two recent innovations: 1) the Metal Anisotropic Reactive Ion etching with Oxidation (MARIO) Process [1], which provides, for the first time, high aspect ratio micromachining capability in bulk titanium; and 2) the High Aspect Ratio Laminated Metal Micromachining (HARLMM) Process, which allows for creation of complex, multilayer titanium devices and heterointegration of these devices with dissimilar material substrates. This work represents the first demonstration of the MARIO and HARLMM Processes in a device application. Details on the fabrication of the device are presented illustrating the simplifications made possible by these two processes and additional simplifications made possible by the single-mask process used to create the sloping electrode structure.
\end{abstract}

\section{INTRODUCTION}

The advent of high aspect ratio bulk micromachining has provided the opportunity for extension of microfabrication capability into the third dimension well beyond that possible using surface micromachining. This has enabled the development large, rigid, highly planar MicroElectroMechanical Systems (MEMS) with highly predictable and reproducible mechanical response. However, material selection in high aspect ratio micromachining has been limited almost exclusively to single crystal silicon, due primarily to the predominance of Deep Reactive Ion Etching (DRIE) Processes, such as the Bosch Process.

While silicon has proven to be an excellent micromechanical material for many applications, no one material can satisfy the requirements of all applications. Consequently, there exists a need for microfabrication techniques that provide greater materials selection capability for high aspect ratio micromechanical applications. The development of the MARIO Process addresses this need by enabling the fabrication of high aspect ratio structures in titanium.

Titanium has a number of properties that make it an attractive alternative to silicon, one of which is its high fracture toughness, which has implications for enhanced durability, shock resistance, flaw tolerance, and notch insensitivity. Interest in these attributes, as well as many others, has motivated efforts to develop process technology that enables implementation of titanium on the micro-scale. Methods such as surface micromachining [2] and bulk micromachining via wet/electrochemical etching $[\mathbf{3}, \mathbf{4}]$ have been reported, but each suffers from aspect ratio restrictions that limit design flexibility and functionality. The MARIO Process, in contrast, has no such limitation. It allows definition of arbitrarily complex structures with micron-scale features and tall vertical sidewalls in bulk titanium substrates.

The utilization of titanium also provides added benefit with regards to process simplification and design complexity through the HARLMM Process. The high fracture toughness of titanium allows foils as thin as $10 \mu \mathrm{m}$ to be handled and processed without the need for fixation to temporary carriers. This enables the fabrication of complex structures with significant cross-sectional variation though-the-thickness by sequential bonding of thin foils. The HARLMM Process also enables heterogeneous integration of high aspect ratio, multi-level, titanium-based MEMS with dissimilar material substrates, therefore providing considerable flexibility with respect to device design, fabrication, and functionality. Similar operations in silicon are possible, but generally require the use of temporary fixation to prevent damage to the delicate thin silicon layers or donation of thin device layers from Silicon on Insulator (SOI) wafers. These methods can add significant cost and impose undesirable constraints on the device design, fabrication, and packaging.

In this paper, the MARIO and HARLMM Processes are used to fabricate a hybrid micromirror device composed of titanium mirrors that are heterogeneously integrated with underlying fixed silicon sloping electrode structures. The sloping electrode structure is utilized to reduce the drive voltage and is created using a simple single-mask process based on the exploitation of Reactive Ion Etching (RIE)-lag.

\section{THEORY}

Recent work has shown that utilization of non-parallel plate electrode geometries, such as terraced [5] or sloping electrodes $[6,7]$, offers the potential for reduced drive voltage relative to comparable parallel plate designs without compromising angular displacement or switching speed. Figure 1 shows a schematic of a particular sloping electrode geometry in which the inside edge of the sloping electrode is not aligned along the centerline defined by the mirror's axis of rotation (i.e. $x=0$ ), but is instead spaced a finite distance, $x_{0}$, away.

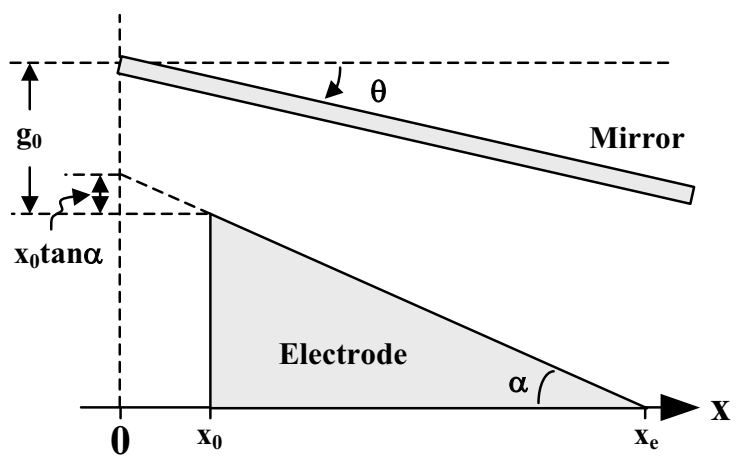

Figure 1. Sloping electrode schematic. Note: only half of the electrode geometry is shown, plane designated by $x=0$ is the symmetry plane.

The electrostatic torque for this electrode geometry can be expressed as 


$$
T_{E}=\int_{0}^{x_{e}} x F_{E}(\theta, x) d x-\int_{0}^{x_{0}} x F_{E}(\theta, x) d x
$$

where $x$ is the moment arm, $F_{E}$ is the electrostatic attraction force per unit length, and $\theta$ is the mirror tilt angle. The first term is the torque applied by a sloping electrode that begins at the centerline and extends out to length $x_{e}$, and the second term subtracts the torque produced by the missing inner portion of the electrode that extends from $x=0$ to $x=x_{0}$. The electrostatic force, $F_{E}$, can be approximated by

$$
F_{E}=\frac{\varepsilon_{0} w V^{2}}{2 g(\theta, x)^{2}}
$$

where $\varepsilon_{0}$ is the dielectric constant, $w$ the width of the mirror, $V$ the applied voltage, and $g$ the effective gap between the mirror and sloping electrode. The effective gap varies as a function of the mirror tilt, position along the $\mathrm{x}$-axis, and the electrode slope, and can be defined as

$$
g=g_{0}-x_{0} \tan \alpha+x \tan (\alpha-\theta)
$$

where $g_{0}$ is the initial gap distance between the highest point on the electrode and the mirror in its rest state (i.e. $\theta=0$ ), and $\alpha$ is the slope angle of the electrode. It should be noted that the above expression does not take into account any vertical 'piston-like' displacement of the mirror. In the current device, such displacement is considered to be negligible due to the resistance to out-of-plane bending afforded by the high aspect ratio torsion springs $(a / b \geq 10)$. It should also be noted that the above analysis does not account for any fringing field effects.

Figure 2 shows a plot of the torque curves produced by evaluation of Eqs. 1-3 for both sloping and parallel plate electrode geometries compared to the restoring torque produced by the torsional springs. The torque enhancement provided by the sloping electrode geometry is clearly evident. For the given case, pull-in voltage is reduced from $147.2 \mathrm{~V}$ for the parallel plate electrode to $57.2 \mathrm{~V}$ for the sloping electrode, representing a $61 \%$ reduction in drive voltage.

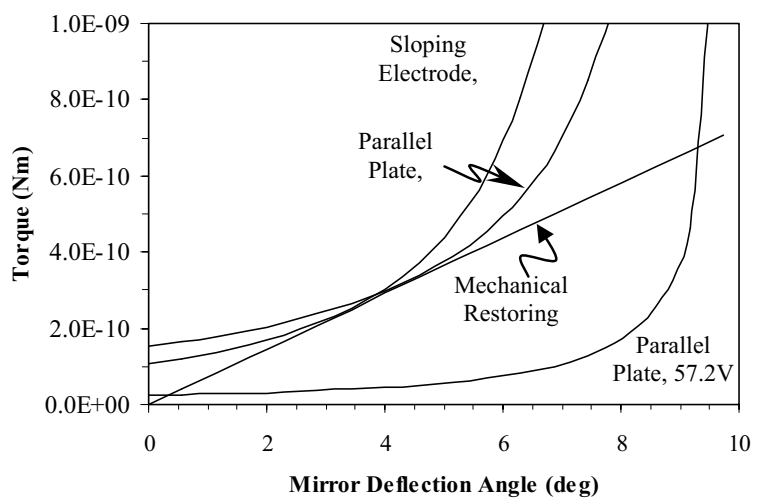

Figure 2. Plot of electrostatic attraction torques for sloping and parallel plate electrodes at varying voltages vs. mechanical restoring torque. Device dimensions used for the calculation were: $100 \times 100 \times 10 \mu m$ mirror; $1 \times 10 \times 60 \mu m$ springs; $x_{0}=5 \mu m ; x_{e}=$ $49.24 \mu \mathrm{m} ; \alpha=10^{\circ}$; and $g_{0}=0.88 \mu \mathrm{m}$.

\section{EXPERIMENTAL DETAILS}

As illustrated in the previous section, the sloping electrode geometry holds promise for significant reduction of drive voltage. Fabrication of structures with such surface gradient topography is by no means simple, however. Methods reported in the literature for fabrication of sloping electrode micromirror devices [5-7] rely on rather complex fabrication routes that could conceivably have detrimental implications on performance, cost, manufacturability, and/or reliability. In this section we present a fabrication method that is far simpler to implement.

The micromirror device is composed of two discrete die, the Electrode Die and the Mirror Die, that are fabricated separately then bonded together in the final step using gold thermocompression bonding. Both die are fabricated via simple, single-mask bulk micromachining processes.

The Electrode Die is fabricated in single crystal silicon substrates using a single-mask, DRIE-based process flow that relies on the phenomena of RIE-lag to simultaneously define all structures on the die. The process begins with lithographic patterning of the silicon wafer in which all components of the Electrode Die are defined, including: sloping electrodes; interconnect lines; remote bondpads; bonding frames that set the mirror/electrode gap height; and grounded landing pads that prevent the mirror from physically contacting the drive electrodes during snap down. Figure 3 shows a portion of the mask pattern used to fabricate the Electrode Die.

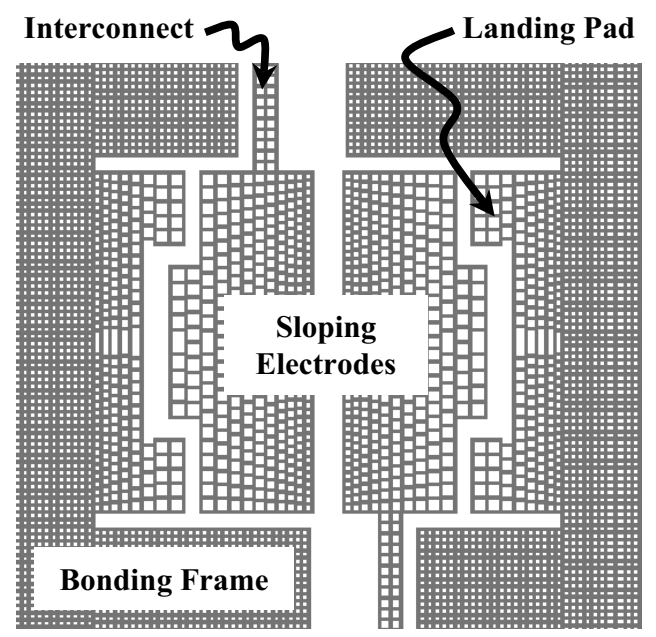

Figure 3. Portion of the Electrode Die mask pattern.

After lithographic patterning the wafer is etched using DRIE, the photoresist mask is stripped, and the wafer is thermally oxidized. The oxidation time is determined by the time needed to oxidize through all thin-walled structures above the etched floors. The oxidized structures are then removed via wet etching in hydrofluoric acid, thus yielding a surface whose topography is defined by the previous DRIE etch depth variation.

Once the HF etch has been performed, all structures on the Electrode Die have been physically defined, however, these structures are not yet electrically active nor isolated from one another. Electrical isolation is achieved by thermally oxidizing the wafer, which isolates the surfaces of the raised structures from the underlying silicon substrate. Electrically active surfaces and connections are then created by blanket gold metallization using ebeam evaporation. The complete process flow for the Electrode Die is summarized in Figure 4. 
a)

\section{Sloping} electrode

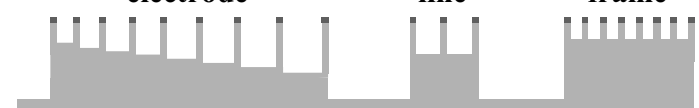

b)

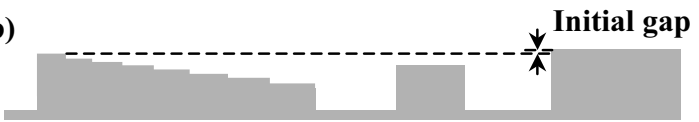

c)

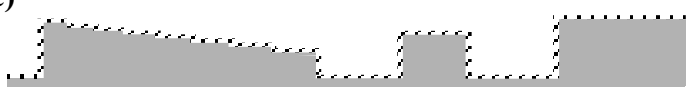

d)

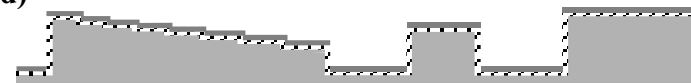

Figure 4. Electrode Die process flow. a) lithographic patterning on single crystal silicon followed by DRIE; b) thermal oxidation followed by HF-based wet etching; c) thermal oxidation; and d) blanket gold metallization.

The Mirror Die is fabricated in free-standing $10 \mu \mathrm{m}$ thick bulk titanium foil which has been chemical mechanical polished (CMP) to facilitate lithographic patterning. A titania etch mask is deposited using reactive sputtering and is patterned using a $\mathrm{CHF}_{3}$ based dry etch. The foil substrate is then deep etched using the MARIO Process, which is a cyclic etch/passivation dry etch recipe consisting of a chlorine-based etch step and a passivating oxidation step. Further details regarding the MARIO Process can be found in reference 1. Etching is carried out until the pattern is completely etched through the foil, resulting in a die with free-standing mirrors supported by high aspect ratio springs. The die is then coated with gold on the backside using e-beam evaporation and finally bonded to the Electrode Die using gold thermocompression at $350{ }^{\circ} \mathrm{C}$ and $\sim 7 \mathrm{MPa}$ applied pressure.

\section{RESULTS \& DISCUSSION}

Figure 5 shows the completed Electrode Die prior to bonding of the Mirror Die. As can be seen, fabrication of complex, 3dimensional structures with multiple levels and sloping surfaces is achieved with a single lithographic masking step. Modulation of the etch via dimensions in the mask pattern causes variation in etch depths across the pattern due to transport limitations imposed by the narrow via dimensions. This etch depth variation is then translated into surface height variation by removal of the thinwalled structures above the etched floors using thermal oxidation and HF-based wet etching.

Utilization of thermal oxidation and wet etching allows for incorporation of both thick- and thin-section structures in the same mask pattern, e.g. the large electrodes and thin interconnect lines of the current device. This provides greater design flexibility relative to other lag-based methods that rely on isotropic etching to remove the structures above the etched floors $[8,9]$. Fabrication of complex structures such as the Electrode Die would not be possible with these methods due to the aggressive undercutting that occurs as a result of isotropic etching.

The use of narrow etch vias for the definition of the device structures causes the etch rate to lag well behind that of the surrounding open areas, therefore defining raised structures with tall vertical sidewalls that stand at varying heights above the substrate floor. Once these structures have been oxidized (Fig. 4c), the vertical sidewalls enable electrical isolation of the upper surfaces of the structures from one another and the surrounding substrate floor due to the poor step coverage of the e-beam metallization (Fig. 4d). Deposition does not occur on surfaces that are parallel to the deposition direction, i.e. the vertical sidewalls, thus electrical isolation is achieved by virtue of the topography of the surface. This therefore enables the definition of all electrically active surfaces without the need for additional patterning steps.

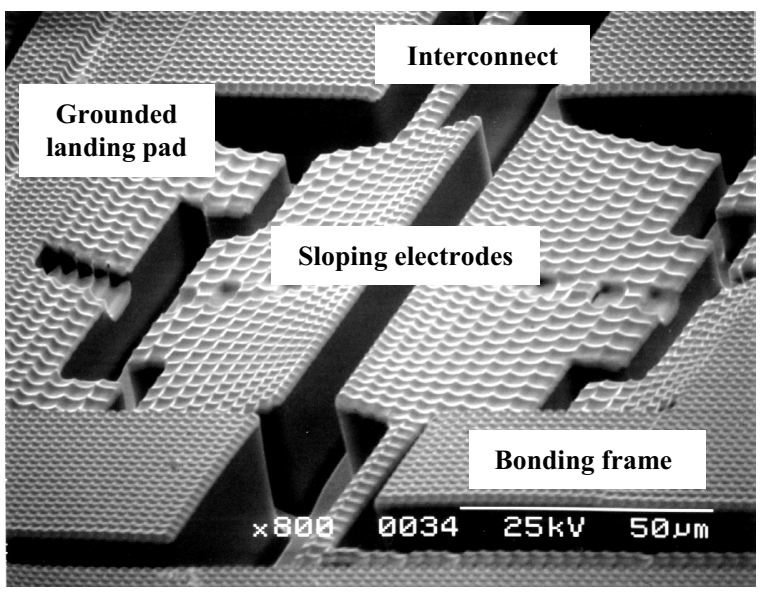

Figure 5. Scanning electron micrograph of the completed Electrode Die prior to bonding of the Mirror Die. The bright regions are gold, while darker regions are thermal oxide. Metallization does not occur on the vertical sidewalls of the structures due to the poor step coverage of the e-beam deposition technique.

The completed micromirror device with the titanium Mirror Die bonded to the silicon Electrode Die is shown in Figure 6. Alignment accuracy is good and the bonded foil shows no apparent signs of deformation, damage, or delamination, therefore indicating that neither bonding pressure nor thermal mismatchinduced residual stresses are a detriment to the current device.
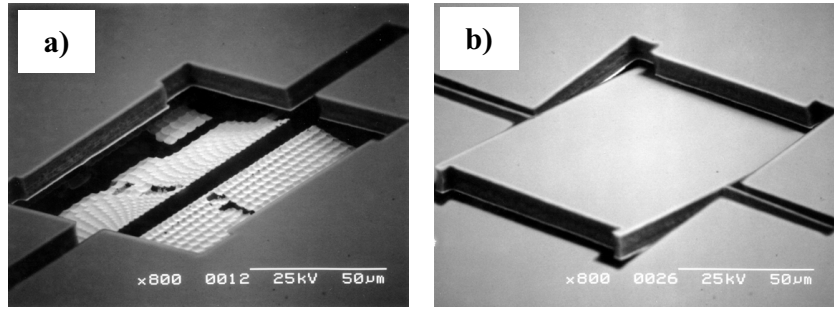

Figure 6. Scanning electron micrographs of the completed hybrid titanium/silicon micromirror device. a) mirror removed to show underlying sloping electrodes with one of the grounded landing pad visible at upper left; b) tilted mirror showing landing tips at corners. The tilted mirror in (b) resulted from stiction after multiple snap down cycles.

Quantitative characterization of the mirror surface quality was carried out using white light optical profilometry. As can be seen in Figure 7, planarity of the mirror surface is rather poor. The average surface roughness of the mirror surface is $95 \mathrm{~nm}$ and the maximum peak-to-valley height differential is $216 \mathrm{~nm}$. The large surface height variation across the mirror prevented accurate measurement of the mirror curvature. Further optimization of the CMP process is currently underway to reduce the roughness. 


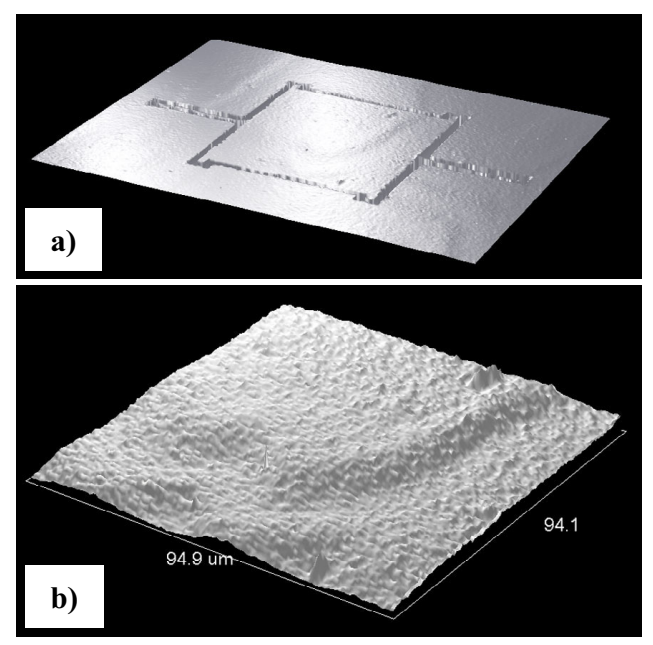

Figure 7. Optical profilometry images of the mirror device. a) image of a single mirror and it's surroundings; and b) close up of the mirror surface itself.

Preliminary dynamic testing of the mirrors shows that intermittent stiction occurs during operation in the digital snapdown mode. This stiction occurs despite the attempt to minimize contact area through the use of narrow landing tips on the mirror, and prevent charging or current-induced welding through the use of complimentary grounded landing pads on the Electrode Die. Stiction in this case most likely results from the presence of gold on both contacting surfaces, therefore it is anticipated that the problem could be remedied to a large extent by removal the gold from the backside of the mirror. However, incorporation of additional strategies, such as application of adhesion-inhibiting surface coatings and novel biasing schemes may be needed as well to ensure complete elimination of stiction.

Operation of the device in the analog scanning mode shows more promising results. Dynamic testing using Laser Doppler Vibrometry, as seen in Figure 8, indicates that pneumatic-damping enables reduction of settling times to well under $1 \mathrm{~ms}$, without resorting to the use of drive signal shaping. These times compare rather favorably to the 1-3 ms settling times often quoted for other large micromirror devices.

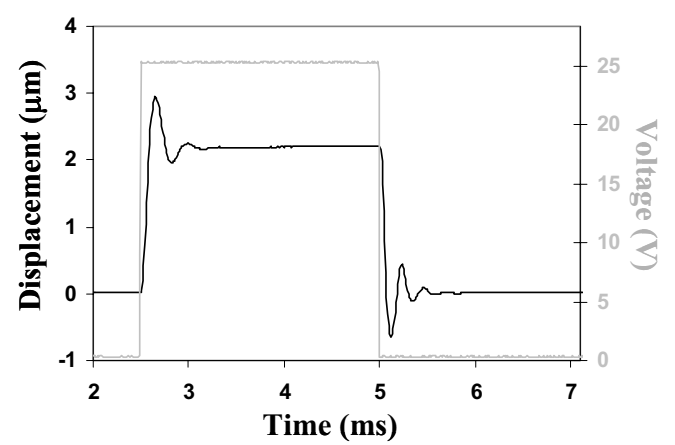

Figure 8. Dynamic response of the mirror device driven in air at $200 \mathrm{~Hz}$ with a $25 \mathrm{~V}$ square wave signal. Resonant frequency is $\sim 4.2 \mathrm{kHz}$ and settling times are well under $1 \mathrm{~ms}$.

\section{CONCLUSIONS}

The simplicity of the process presented here lies in direct contrast to other methods proposed for the fabrication of sloping electrode-based micromirror devices which require: a) the use of costly SOI wafers to provide the thin silicon layer needed for the mirror and springs [5]; b) careful optimization of multiple electrodeposition and planarization steps to develop the desired sloping electrode geometry and flat, low roughness mirrors [6]; or c) thorough optimization of solder pad geometry and location to ensure accurate and consistent reflow-based self-assembly [7]. Utilization of the MARIO, HARLMM, and RIE-lag processes circumvents these issues, providing a potentially simple and robust method for fabrication of high performance micromirror devices.

\section{ACKNOWLEDGMENTS}

The authors would like to thank. G. D. Cole, M. Napoli, and the Materials Technology Office at the Defense Advanced Research Projects Agency.

\section{REFERENCES}

1. M. F. Aimi, M. P. Rao, N. C. MacDonald, A. S. Zuruzi, and D. P. Bothman, "High-Aspect-Ratio Bulk Micromachining of Titanium," Nature Materials, 3, 103 (2004).

2. C. O'Mahony, M. Hill, P. J. Hughes, and W. A. Lane, "Titanium as a Micromechanical Material," J. Micromech. Microeng., 12, 438 (2002).

3. T. N. Pornsin-sirirak, Y. C. Tai, H. Nassef, and C. M. Ho, "Titanium-alloy MEMS Wing Technology for a Micro Aerial Vehicle Application," Sens. Actuators A Phys., 89, 95 (2001).

4. P. -F. Chauvy, C. Madore, and D. Landolt, "Electrochemical Micromachining of Titanium Through a Patterned Oxide Film," Electrochem. Solid St., 2, 123 (1999).

5. R. Sawada, J. Yamaguchi, E. Higurashi, A. Shimizu, T. Yamamoto, N. Takeuchi, and Y. Uenishi, "Single Si Crystal 1024ch MEMS Mirror Based on Terraced Electrode and a HighAspect Ratio Torsion Spring for 3-D Cross-Connect Switch," 2002 IEEE/LEOS International Conference on Optical MEMS: Optical MEMS 2002, Lugano, Switzerland, 8/20-23/02, IEEE Laser and Electro-Optics Society, Piscataway, NJ (2002), pp. 11-12.

6. K. Reimer, R. Engelke, M. Witt, and B. Wagner, " $16 \mathrm{k}$ Infrared Micromirror Arrays with Large Beam Deflection and Tenth Millimeter Pixel Size," Proceedings of the SPIE Conference on Miniaturized Systems with Micro-Optics and MEMS, Vol. 3878, Santa Clara, CA, 9/20-22/99, The International Society of Optical Engineering, Bellingham, WA (1999), pp. 272-90.

7. B. McCarthy, V. Bright, and J. Neff, "A Multi-Component Solder Self-Assembled Micromirror," Sens. Actuators A Phys., 103, 187 (2003).

8. T. Bourouina, T. Masuzawa, and H. Fujita, "MEMSNAS: Microloading Effect for Micromachining 3D Structures with Nearly Arbitrary Shapes: Application to Micro-optics on Silicon," 2001 IEEE/LEOS International Conference on Optical MEMS: Optical MEMS 2001, Okinawa, Japan, 9/25-28/01, IEEE Laser and Electro-Optics Society, Piscataway, NJ (2001), pp. 81-82.

9. T. -K. A. Chou and K. Najafi, "Fabrication of Out-of-Plane Curved Surfaces in Si by Utilizing RIE-Lag," $15^{\text {th }}$ IEEE International Conference on Micro Electro Mechanical Systems MEMS 2002, Las Vegas, NV, 1/20-24/02, IEEE, Piscataway, NJ (2002), pp. 145-148. 